\title{
Computer simulation: A case of conflict
}

\author{
JAMES E. SPIVEY and PAMELA JACKSON-SMITH \\ University of Kentucky, Lexington, Kentucky 40506
}

\begin{abstract}
Herein is described an 18-month adventure involving the translation to computer-aided instruction (CAI) format of a traditional module that had been successfully used for years in an experimental psychology course. The primary focus of the paper is on the lessons learned during this transition. The major problems of translation that were encountered are discussed, as are unanticipated, often seemingly trivial, human-factors problems that were the most troublesome, and, consequently, the most educational.
\end{abstract}

For years, our experimental psychology laboratory course was taught in the standard fashion. Students performed a variety of "canned" experiments using each other as subjects, analyzed the data, and wrote up an APA-style paper based on each experiment. Care was taken to insure that the experiments represented a wide range of psychological areas, experimental methods, and research designs. In spite of the usual complaints that one typically hears about research methods courses (Spivey, 1983), the course was well rated and a joy to teach.

When the psychology department secured a large CAUSE (comprehensive assistance to undergraduate science education) grant from the National Science Foundation (NSF), the department embarked on a great number of ventures into educational reform. The most optimistic reformers of the group, of course, were those who were committed to CAI. We soon found, as do most reformers, that enthusiasm needs to be tempered with infinite patience and constantly exposed to reality testing. A case in point is the following scenario involving an extremely successful traditional module that, it was agreed, could be improved through a simple conversion into a CAI format.

At one point in the experimental psychology course, within-subjects designs are discussed. In the laboratory, an attempt is made to reinforce and apply knowledge

The CAI activities described in this paper are supported by National Science Foundation Grant SER-8005325, which is concerned with the more general task of upgrading the psychology department's undergraduate program. Ronald C. Dillehay is director of the grant; Arthur J. Nonneman served as acting director in Dillehay's absence during the initial year of the grant and contributed substantively to the development of several CAI modules. The contributions of James R. Sanders and Daniel L. Stufflebeam as external evaluators and of Timothy A. Smith, Craig J. Petrun, and Isabella M. Zembrodt as internal evaluators are appreciated. Programming was performed by John A. Walker; systems advisors were Richard G. Davis and David C. Lowe. Sole responsibility for statements contained in this paper lies with James E. Spivey and Pamela Jackson-Smith. Requests for reprints or additional information should be sent to James E. Spivey, Department of Psychology, Kastle Hall, University of Kentucky, Lexington, Kentucky 40506. that students have gained from the text and lectures. For these purposes, we developed a few years ago a module concerned with "conflict."

Projected onto a wall screen were triangles and/or circles. The students, seated at tables, were told to move a toggle switch so as to approach triangles and avoid circles. For example, if a triangle appeared on the left of the screen, the toggle switch was to be moved to the left. For the first 28 trials, all subjects received identical training. The slide employed on the last trial, Trial 29, was the test slide that defined the group. To mention two of the possible groups, the approach-approach group saw a triangle on each side of the screen, and the approach-avoidance group saw a triangle and a circle on the same side of the screen. The experiment could have been run either as a between- or as a within-subjects design. The latter was chosen, and order effects were controlled by having one-half of the students receive the approach-approach conflict first and the other half of the students receive the reverse order. ${ }^{1}$

Students greatly enjoyed the module, and it served as an excellent concrete example of the problems, and their appropriate solutions, found in research with this paradigm. Statistical significance was a certainty, with mean response latencies for Trial 29 averaging, for example, $4.99 \mathrm{sec}$ for the approach-approach condition and $12.64 \mathrm{sec}$ for the approach-avoidance condition.

Several advantages were seen in "computerizing" the module. These included freeing equipment and a room that was tied up for a week each semester with wires running everywhere and avoiding the pesky, although simple, electronics work. The greatest advantage was the automatic collating of data. With the traditional format, data dissemination was time-consuming and boring. Either the teaching assistant assumed the responsibility for making "master" data sheets with the individual trial-by-subject values nestled in their appropriate cells, or each student wrote his/her score on the board, after which all students copied everyone's data for later analysis. There had to be a better way.

The awarding of the CAUSE grant and the subsequent purchase of Apple II-Plus microcomputers, eight of which were tied to a Winchester hard-disk system, 
made possible this better way (Lowe, 1983). So, we thought we would just put the thing on the computer that week. Eighteen months later, we finally have a module that may not perform quite as well as the handson version but promises to do so with a few more weeks of work. From the problems encountered in this quest for the optimal module, a great deal has been learned. Some of these lessons will now be made explicit.

\section{PROBLEMS OF TRANSLATION}

We never realized how much a teacher communicates with his/her hands until we began to write instructions for this module. The traditional module was well understood by students, but the first two versions of the computer module were criticized by students for unclear instructions. Several elements were missing. When the traditional module was employed, the instructor gestured, pointed, and even demonstrated with a sample apparatus (toggle switch). Students were allowed to ask questions, many of which were unpredicted by the instructor, and were made to feel confident of their ability to perform the task prior to its initiation. When the instructions were written for the computer module, the instructors/programmers suffered from an embarrassing failure of transfer of training. That is, the variety and quality of instructional techniques used for the traditional module were not employed with the CAI version.

We now realize that one must spend a great deal of time on instructions, including the pilot-testing phase, and that this time will exceed by several multiples the time allotted by novice programmers. These instructions must contain several elements. Besides obvious criteria such as clarity and completeness, the mode of expression must not be too formal or impersonal. The machine, threatening to many students, is replacing a warm, smiling, personable instructor who provides eye contact and reassurance-a tough act to follow. The second element needed is a set of concrete examples involving not only the training stimuli, but also responses by the student, which are followed by feedback. Another element is the student's option to have the instructions and examples repeated by the machine.

A fourth element is the presence of an instructor who can answer unanticipated questions; some students are amazingly creative in misconstruing instructions. A final element is the movement of the instructor about the room to insure that everyone is following directions. Some students, although they understand the instructions, insist on doing things another way. In a laboratory in which other students are depending on these data to test a hypothesis, slight procedural deviations can introduce an array of extraneous variables. Many people who advocate CAI state that a major advantage is their true "stand-alone" capability, with no need for the presence of an instructor. While this is certainly true of many types of modules, particularly those concerned with remediation, some modules are better used in the presence of an instructor. In fact, a friendly presence, especially early in the semester, boosts student morale and facilitates learning.

A final word about instructions, and computer modules in general, relates to their two-dimensional character. Rotating something in space for a class, or having students view an apparatus from several perspectives can be very difficult to elucidate in two dimensions. This observation is relevant, of course, only to those without funds for high-technology graphics.

\section{PROBLEMS OF HUMAN ENGINEERING}

When the module was translated, students needed some way of responding "left" or "right." For this purpose, two disparate, easily accessible keys were selected, and students were asked to press " $\mathrm{s}$ " for left and "l" for right. We knew that the word "left" begins with an "l," but no confusion was found with pilot sub. jects, so no problems were anticipated. From this experience, three lessons were learned. The first is a corollary of Murphy's Law. This corollary states that any small contradiction or inconsistency in a module not only will be glaringly obvious to students but will become for some "dd" (or "Dd") types an insurmountable obstacle. Another lesson learned is that people have much higher standards for computer modules than for traditional ones. Students tolerate inadequacies in traditional modules and see the instructor as doing the best he/she can under the circumstances. However, these same students demand perfection from CAI, perhaps because of the absence of a personal relationship or because they believe the machine has the potential to be perfect, whereas a person does not. These expectations must be considered when traditional teaching methods are compared with CAI.

The third lesson learned relates to the validity of pilot data. Although pilot data are often useful, we continue to find that the most valid data in testing the modules, and sometimes the only valid data, come from the students in the course. The ideal way to test a module is to present it to the class as a regular exercise, to ask them to work in pairs, and to "exteriorize their minds." That is, they are asked to express aloud anything that is going on in their minds. They also are asked to continually discuss aloud the module with their partner. One evaluator per two or three pairs of students listens to and records this verbalizing. After the module is completed, the lecture and laboratory teachers, all evaluators, and the laboratory students hold a debriefing session to discuss problems and possible improvements in the module. This great effort actually saves a lot of time. The "case history" portion, in which students externalize their thoughts, is especially valuable, in part because the students soon forget many of their early impressions. 


\section{THE JOY OF CONFLICT}

In casting about for a solution to the key-designation problem, it seemed that what really was needed was something more akin to the original situation (the toggle switch). So the programmer, a man of infinite patience, was told that the program was to be rewritten, again. The new module employed a joystick, the position of which was indicated by a cursor visible on the screen. This new version had the advantage of allowing self-correction (retracing) prior to the completion of the response and was more time-consuming. The latter factor is important because, with the single-buttonpress response, many students either do not take the response seriously or do not take time to think about their response; they seem to concentrate too much on pure response speed. Some even press both buttons at the same time in an obvious effort to speed through the module. The buttonpress data, even for those students who were not baffled by key designation, never indicated significant differences between groups.

The joystick innovation taught us something about this hardware and its limits. That is, it is not possible for the Apple to keep track of the position of the joy. stick with a visual cursor in the lower portion of the monitor while timing the interval between the presentation of the stimuli (triangle or circle) and the arrival of the joystick at either the extreme left or right position. It finally was determined that the task set for the system required external timing devices, one for each machine, for which money was not available.

The joystick cul de sac, although embarassing, could not have been avoided. No one on the staff has had prior experience with this systern, and even the advisors have had to learn as they go. The lesson here is one of budgeting; funds for peripheral equipment and programming are usually exhausted much faster than anticipated.

The present solution, which is being tested with students now in the class, is to return to the keypress response using " $z$ " for left and "//" for right. The response required is five keypresses (FR 5) instead of one (FR 1). These two modifications should obviate, respectively, the semantic problem of key designation and the problems often associated with brief, low-effort responses. The FR component also will allow selfcorrection and involve the learner in more contact with each trial.

\section{SUCCESS, FAILURE, REGRETS}

The design goals of the module have been met, and more sophisticated instrumentation has led to greater control over the sequencing and timing of stimulus materials. Students also are able to progress at their own rate, which they seem to appreciate. Further, the module is extremely popular. The students especially appreciate the use of the Winchester hard-disk system that organizes the data from the various Apples into a subjects-by-trials matrix, which is then printed out. ${ }^{2}$ Students are allowed to use the statistical tests written for the Apple, although early in the course they are required to perform tests manually to guarantee an understanding of statistical procedures.

The only problem with the module is that it still does not provide valid data-no small problem. The continued use of this module in its automated version depends on the success of the FR modification or the securing of funds for external timing equipment. But what if the CAI module, even if it produces valid data, turns out to be no better than the traditional module as measured by both student interest and learning (see Belmore, 1983)? Can we conclude that a lot of time and money have been wasted? No, we cannot, for obvious reasons expressed in the preceding paragraph and for some not-so-obvious reasons now to be explicated. Our reluctance to return to the traditional version of the module is based not merely on a need to justify our efforts but, rather, on the belief that in the years ahead students will show an increasing impatience not only with sloppy stimulus control but, even more, with pedestrian tasks such as data collation. This intolerance will be shared by future teaching assistants. The professor in charge of the course will be even less tolerant, especially after being exposed to a better way.

We have only one regret resulting from long involvement with this module, and that is that we were not more conversant with the hardware at the start (see Kieras, 1981). However, one of the great accomplishments of the CAUSE grant has been to educate faculty, even more than students, in the use of computers. Considering our date of entry into this project, it is not surprising that difficulty was encountered in locating competent consultants. Those working in more cosmopolitan areas of the nation, wherein computing is more integrated into academic life, may have difficulty appreciating our plight. The enormous differences in technological sophistication that exist across this country lead to two different conclusions about where grant monies should be directed. We hold one view, primarily because we feel that the receipt of this grant has served as a highly fertile seed in our community. The department is being used as a model for attempted changes in other departments. One-fourth of the enrollment of the experimental psychology course consists of computer science majors; another one-fourth are from other departments, mainly business, and the service role of the course continues to grow. CAUSE staff are conducting workshops and consulting in the public schools and in the community. They now are serving, as a result of their CAUSE experience, as the long. needed $\mathrm{CAI} / \mathrm{microcomputer}$ consultants for the community. 


\section{REFERENCES}

Belmore, S. M. RELEASE FROM PI: Comparison of traditional and computer modules in an experimental psychology laboratory. Behavior Research Methods \& Instrumentation, 1983, 15, 191-194.

KIERAS, D. E. Effective ways to dispose of unwanted time and money with a laboratory computer. Behavior Research Methods \& Instrumentation, 1981, 13, 145-148.

Lowe, D. C. A Winchester hard-disk integrated computer-assisted instructional laboratory: Hardware and data management considerations. Behavior Research Methods \& Instrumentation, 1983, 15, 181-182.

SPIVEY, J. E. Software development for computer-assisted instruction in experimental psychology. Behavior Research Methods \& Instrumentation, 1983, 15, 183-186.

\section{NOTES}

1. For those familiar with conflict research, it should be indicated that when a between-subjects design was employed, a third, no-conflict, group was included (on Trial 29 a triangle was presented on one side and a circle on the other). When the within-subjects design was employed, the three-condition model was too cumbersome and time-consuming if done properly, so some control was sacrificed for clarity.

2. Pedagogues will appreciate the fact that for two laboratory modules the manual collating of data is still required. We find that students make certain errors of collation, not due to simple carelessness, which indicate that they need some manual experience for the design and data handling lessons to be fully understood. Furthermore, this manual collation is a skill that researchers probably will need for at least several more years. 